\title{
Using a Dynamic Interval Type-2 Fuzzy Interpolation Method to Improve Modeless Robots Calibrations
}

\author{
Ying Bai ${ }^{1}$ and Dali Wang ${ }^{2}$ \\ 1. Department of Computer Science and Engineering, Johnson C. Smith University, Charlotte 28216, USA \\ 2. Department of Physics and Computer Science, Christopher Newport University, Newport News 23606, USA
}

\begin{abstract}
This paper is an extended research for a novel technique for the position error compensations of the robot and manipulator calibration process based on an IT2FEI (interval type-2 fuzzy error interpolation) method. Traditional robots calibration implements either model or modeless method. The compensation of position error in modeless method is to move the robot's end-effector to a target position in the robot workspace, and to find the target position error based on the measured neighboring 4-point errors around the target position. A camera or other measurement device is attached on the robot's end-effector to find and measure the neighboring position errors, and compensate the target position with the error interpolation results. By using the IT2FEI technique provided in this paper, the accuracy of the position error compensation can be greatly improved, which has been confirmed by the simulation results given in this paper. Compared with some other popular traditional interpolation methods, this IT2FEI technique is a better choice. The simulation results show that more accurate compensation result can be achieved using this technique compared with the type-1 fuzzy interpolation method.
\end{abstract}

Key words: Modeless robots calibrations, position error compensations, interval type-2 fuzzy interpolations, dynamic online fuzzy interpolation algorithm.

\section{Introduction}

The prerequisite requirement of the robotic modeless calibration is the successful self-calibration of the camera $[1,2]$. Both internal and external parameters of the camera need to be calibrated accurately [3, 4]. Then modeless robot calibration is divided into two steps [5]. The first step is to identify the position errors for all grid points on a standard calibration board, which is installed on the robot's workspace. A calibrated camera or other measurement device is attached on the robot's end-effector to find the neighboring position errors. This process can be considered as an identification process, which is shown in Fig. 1.

At each grid point, a calibrated camera is used to check the position errors of the end-effector of the robot. In Fig. 1, the desired position of the grid point 0

Corresponding author: Ying Bai, Ph.D., professor, research fields: intelligent and fuzzy controls, robots calibrations. is $\left(x_{0}, y_{0}\right)$, and the actual position of the robot end-effector is $\left(x_{0}^{\prime}, y_{0}^{\prime}\right)$. The position errors for this grid point are $e_{x}=x_{0}-x_{0}^{\prime}$, and $e_{y}=y_{0}-y_{0}^{\prime}$.

The robot will be moved to all grid points on the standard calibration board, and all position errors on these grid points will be measured and stored in the memory for future use.

In the second step, the robot's end-effector is moved to a target position that is located in the range of the workspace. The target position error could be found by an interpolation technique using the stored 4-neighboring grid position errors around the target position, which were obtained from the first step. Finally, the target position could be compensated with the interpolation results to obtain more accurate positions.

Triantafilis [6] and Suzana et al. [7] reported approaches of using fuzzy interpolation methods to estimate the soil layer and geographical distributions for GIS database. Song et al. [8] described a fuzzy logic methodology for 4D (4-dimensional) systems 


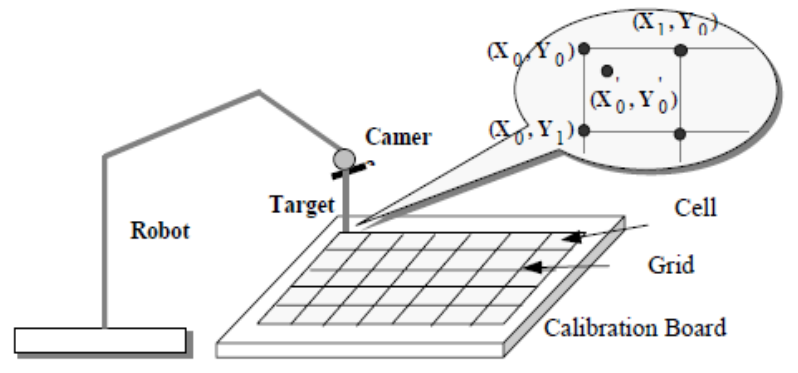

Fig. 1 Setup of the robotic modeless calibration.

with optimal global performance using enhanced cell state space. The most popular interpolation techniques applied in the position compensations of the modeless robotic calibration include the bilinear interpolation and cubic spline interpolation methods; both methods can achieve satisfactory interpolation results for general calibration process [2]. Since the actual position errors are randomly distributed, and it is impossible to pinpoint a position on the error surface at any given moment, the traditional interpolation techniques are unable to provide an accurate estimation of the position errors. The IT2FEI (interval type-2 fuzzy error interpolation) technique utilizes an interval type-2 fuzzy inference system to estimate the position errors, which is consistent with the random distributed nature of position errors. The position errors can be considered as a fuzzy set at any given moment of the time. The fuzzification process takes into account of a range of errors rather only a crisp error value. Therefore, the fuzzy error interpolation technique has the fundamentals to improve error estimation results.

The interval type-2 fuzzy inference is the process of formulating the mapping from a given input to an output using interval type-2 fuzzy logic $[9,10]$. The mapping then provides a basis from which decisions can be made, or patterns discerned. Interval type- 2 fuzzy inference systems have been successfully applied in fields such as automatic controls, data classification, decision analysis, expert systems, and computer vision [9]. Aminifar and Marzuki [11] reported an analysis about the uncertainty between the type-1 and IT2FLS (interval type-2 fuzzy logic system). Chang [12] presented a new method for handling fuzzy rule interpolation in sparse fuzzy rule-based systems based on interval type-2 fuzzy sets. Kashyap [13] reported a research of using an interval type-2 fuzzy logic system to perform image fusion. Lu et al. [14] reported to control a nonlinear system with the interval type-2 FLC. Wu and Mendel [15] reported a new method to simplify the design process for the interval type-2 fuzzy system. Schrieber and Biglarbegian [16] reported to use an interval type-2 FLC to control the FPGA production process. Tsai et al. [17] reported to use an interval type-2 FLC to control an intelligent bike. Nurmaini and Tutuko [18] described a motion coordination of swarm robots using IT2FLC (interval type-2 fuzzy logic controller) to control swarm robots coordination to produce smooth trajectory without collision. Mendel et al. [19] discussed and analyzed different structures of type-1 and type-2 fuzzy controllers. Kumbasar [20] examined the robust stability of a PD type Single input SIT2-FLC structure. All of these updated applications provide a prospective future for interval type- 2 fuzzy inference system.

This paper is organized in five sections. After this introduction section, the operational principles of the type-1 fuzzy interpolation technique are provided in section 2. Section 3 discusses the interval type-2 fuzzy error interpolation method. A simulation is given in section 4 to illustrate the effectiveness of the fuzzy error interpolation technique. Section 5 presents the conclusion.

\section{Type-1 Fuzzy Error Interpolation System}

\subsection{The On-line Versus Off-line Fuzzy System}

In order to improve the compensation accuracy, a dynamic on-line fuzzy error interpolation method is introduced. The traditional fuzzy inference system uses pre-defined membership functions and control rules to construct lookup tables; then a control output is selected from the lookup table. This type of system is called off-line fuzzy inference system because all 
inputs and outputs have been defined prior to the application process. The off-line fuzzy system cannot meet our requirement for the several reasons. First, the position error of the target point is estimated based on four errors of the neighboring grid points, and these four neighboring errors are randomly distributed. The off-line fuzzy output membership functions are defined based on the range of errors, which is the neighboring errors' range. However, this range estimation is not as accurate as the real errors obtained on the grid points. Second, since each cell needs one lookup table for the off-line fuzzy system, it would require a huge memory space to save a large number of lookup tables. This results in demanding requirement in both space and time, and as a result, becomes not practical for real time processing. For example, in our study, $20 \times 20$ cells are utilized on the calibration board (each cell is $20 \times 20 \mathrm{~mm}$ ); this would require 400 lookup tables. By using an on-line dynamic fuzzy inference system, the target position error can be estimated by combining the output membership functions, which are defined based on the real errors on neighboring grid points and the control rules in real time. The output membership functions are not predetermined, and their definitions are based on the real errors on the grid points, not a range.

Fig. 2 shows the definition of the fuzzy error interpolation inference system.

Each square that is defined by four grid points is called a cell; and each cell is divided into four equal smaller cells, which are NW, NE, SW and SE, respectively (Fig. 2a). The position error at each grid point is defined as $\mathrm{P}_{1}, \mathrm{P}_{2}, \mathrm{P}_{3}$ and $\mathrm{P}_{4}$.

For the fuzzy inference system, we apply the fuzzy error interpolation method in two dimensions separately, so the inputs to the fuzzy inference system are $e_{x}$ and $e_{y}$ and the outputs are $e e_{x}$ and $e e_{y} \quad$ (Fig. 2b). The control rules are shown in Fig. 2c, and are discussed following the discussion of membership functions.

\subsection{Membership Functions}

In this work, the distance between two neighboring grid points on the standard calibration board is $20 \mathrm{~mm}$ in both $x$ and $y$ directions, which is a standard value for a mid-size calibration workspace. The calibration board includes a total of 20 by 20 cells, which is equivalent to a 400 by $400 \mathrm{~mm}$ space.

The input membership functions for both $x$ and $y$ directions and the predefined output membership functions are shown in Fig. 3. The predefined output membership functions are used as default functions, and the final output membership function will be obtained by shifting the default one by the actual error values on the grid points.

The gaussian-bell waveforms are selected as the shape of the membership functions for both inputs (Fig. 3a) in $x$ and $y$ directions. The ranges of inputs are between $-10 \mathrm{~mm}$ and $10 \mathrm{~mm}$ (20 $\mathrm{mm}$ intervals). Zhuang and $\mathrm{Wu}$ [21] reported a special histogram method to estimate the optimal membership function distribution. However in our case, a gaussian-bell shape is selected due to the fact that most errors in real world match this distribution. We use $\mathrm{W}$ and $\mathrm{E}$ to

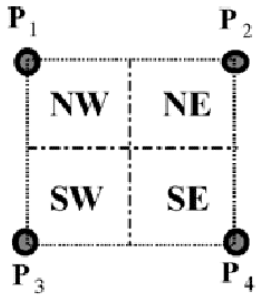

(a)

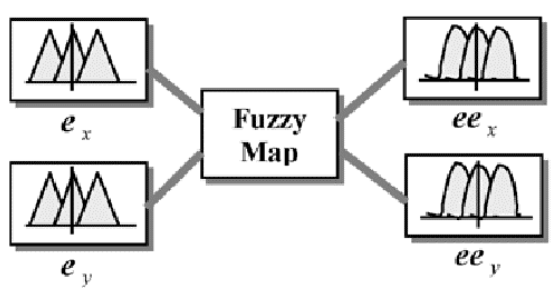

(b)

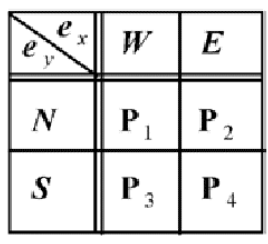

(c)

Fig. 2 Definition of the fuzzy error interpolation system. 


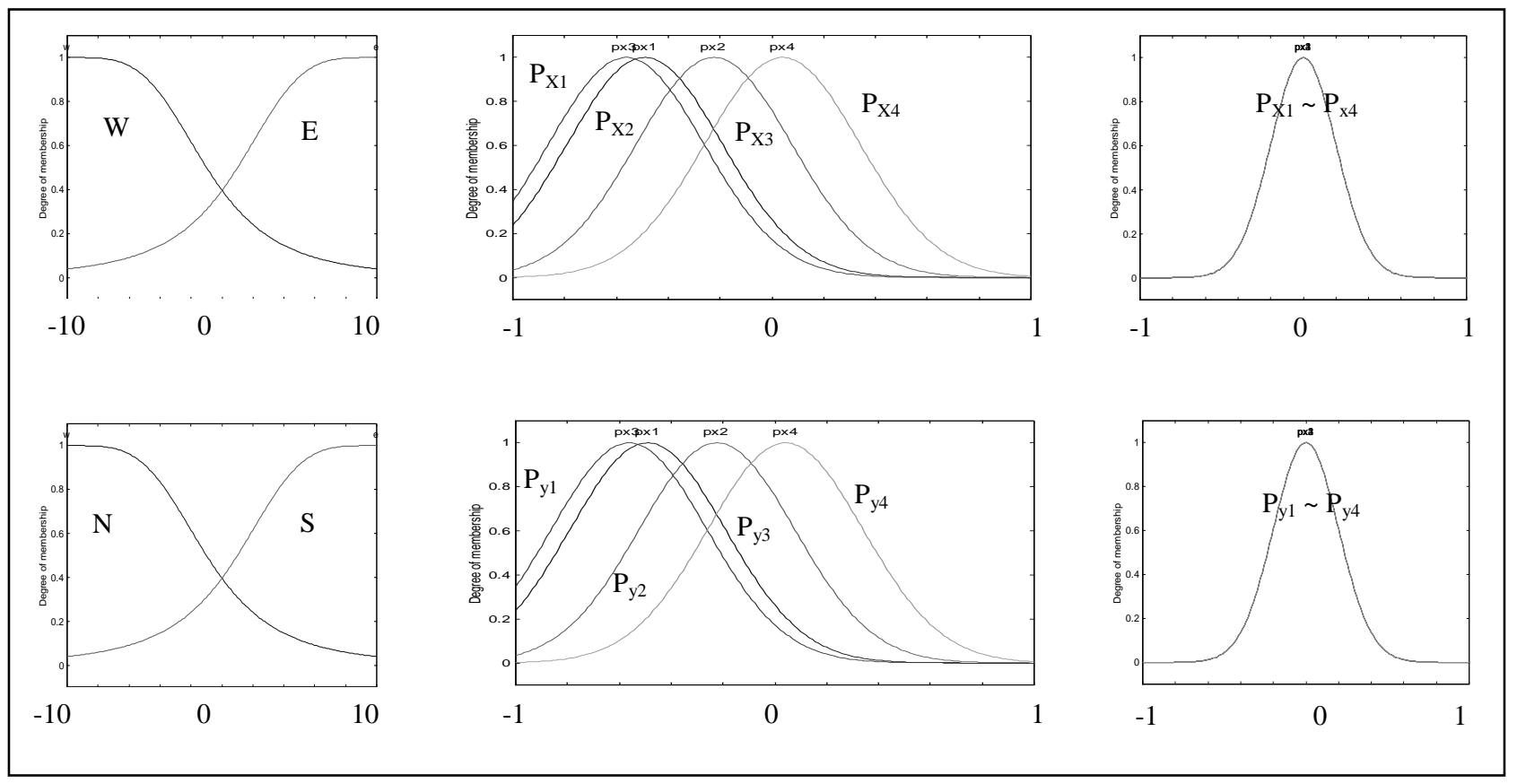

(a)

(b) (c)

Fig. 3 Input and output membership functions.

represent the location of inputs in $x$ direction, $\mathrm{N}$ and $\mathrm{S}$ to represent the location of inputs in $y$ direction. Unlike the traditional fuzzy inference system, in which all membership functions should be determined in advance to produce the lookup table, the output membership functions will be determined during the application of the fuzzy inference system in real time. This is called dynamic fuzzy system.

Fig. 3b shows an example of the output membership functions, which are related to the simulated random errors at neighboring grid points. Each $\mathrm{P}_{x i}$ and $\mathrm{P}_{y i}$ correspond to the position error at the $i$ th grid point in $x$ and $y$ directions, respectively. During the design stage, all output membership functions are initialized to a gaussian waveform with a mean of 0 and a range between $-0.5 \mathrm{~mm}$ and $0.5 \mathrm{~mm}$, which is a typical error range for this workspace in robotic calibration (Fig. 3c) [5]. These output membership functions will be determined based on the errors of the neighboring grid points around the target in the workspace as mentioned above. For example, during the compensation process if the input position in the $x$ direction is in the NW area of a cell, the associated output membership function should be modified based on the position error in the NW grid point $\mathrm{P}_{1}$. This modification is equivalent to shift the $\mathrm{P}_{\mathrm{x} 1}$ Gaussian waveform (Fig. 3b) and allow the center of that waveform to be located at $x_{0}=$ the position error value of the $\mathrm{P}_{1}$ in the $x$ direction. Similar modification should be performed for the position error in the $y$ direction. It can be seen from Fig. 3b that the performance loss would be significant if the default output membership function is utilized, which is shown in Fig. 3c, for the position compensation process.

\subsection{Control Rules}

The control rules shown in Fig. 2c can be interpreted as follows after the output membership functions are determined:

If $e_{x}$ is $\mathrm{W}$ and $e_{y}$ is $\mathrm{N}, e e_{x}$ is $\mathrm{P}_{x 1}$ and $e e_{y}$ is $\mathrm{P}_{y 1}$ (NW).

If $e_{x}$ is $\mathrm{W}$ and $e_{y}$ is $\mathrm{S}, e e_{x}$ is $\mathrm{P}_{x 3}$ and $e e_{y}$ is $\mathrm{P}_{y 3}$ (SW).

If $e_{x}$ is $\mathrm{E}$ and $e_{y}$ is $\mathrm{N}, e e_{x}$ is $\mathrm{P}_{x 2}$ and $e e_{y}$ is $\mathrm{P}_{y 2}$ (NE). 
If $e_{x}$ is $\mathrm{E}$ and $e_{y}$ is $\mathrm{S}, e e_{x}$ is $\mathrm{P}_{x 4}$ and $e e_{y}$ is $\mathrm{P}_{y 4}$ (SE).

Each $\mathrm{P}_{i}$ should be considered as a combination of two error components, $\mathrm{P}_{x i}$ and $\mathrm{P}_{y i}$, which are corresponding to errors in both $x$ and $y$ directions. The error on NW grid point should take more weight if the target position (input) is located inside the NW area on a cell. Similar conclusion can be derived for errors on SW, NE and SE grid points.

\section{Interval Type 2 Fuzzy Interpolation System}

3.1 Overview of the Interval Type 2 Fuzzy Interpolation System

Similar to type 1 fuzzy inference system, the type 2 fuzzy inference system still uses the input and output membership functions, combined with the control rules, to derive the outputs $[10,15]$. However, the fuzzy sets used in the type 2 fuzzy logic or the membership grades involved in each membership function are not crisp values, but another fuzzy sets. This means that the membership degrees for all membership functions used in the type 1 fuzzy system are fixed values and can be determined uniquely before the fuzzy inference system works. But the membership degrees for all membership functions used in the type 2 fuzzy system are fuzzy sets. The difference between the standard type-2 fuzzy system and the so-called interval type-2 fuzzy system is that in the former system, the membership degrees are pure fuzzy sets, but the membership degrees are a set of crisp values with a range of $0 \sim 1$ or an interval for the latter.

Fig. 4 shows the functional block diagram of an interval type-2 FLS [22]. It is similar to type-1 FLS, but the major difference is that at least one of the fuzzy sets in the rule base is an IT2 fuzzy set. The outputs of the inference engine are IT2 fuzzy sets, and a type-reducer is needed to convert them into a type-1 fuzzy set before defuzzification can be started.

Some fundamental operations in the type-2 fuzzy

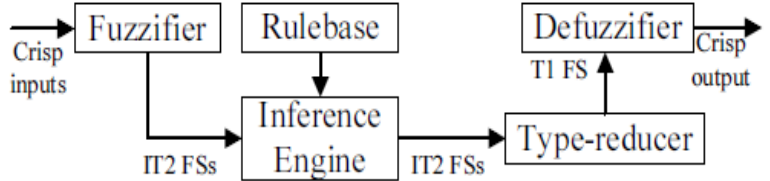

Fig. 4 A functional block diagram of the interval type-2 fuzzy system.

system are union (1), intersection (2) and complement (3) [23]. The union for interval type-2 fuzzy sets $\tilde{A}$ and $\tilde{B}$ is:

$$
\begin{aligned}
& \tilde{\widetilde{A}} \cup \widetilde{\widetilde{B}}=\left\{\int_{x \in X} \mu_{\tilde{\tilde{A}}}(x) \cup \mu_{\tilde{\tilde{B}}}(x) / x\right\}= \\
& \left\{\int_{x \in X}\left[\begin{array}{c}
\int_{\alpha \in\left[\underline{\mu}_{\tilde{A}(x)} \vee \underline{\mu}_{\tilde{B}(x)}, \bar{\mu}_{\tilde{A}(x)} \vee \bar{\mu}_{\tilde{B}(x)}\right.} 1 / \alpha \\
\end{array}\right] / x\right\}
\end{aligned}
$$

The intersection for interval type-2 fuzzy sets $\tilde{A}$ and $\tilde{B}$ is:

$$
\begin{aligned}
& \tilde{\widetilde{A}} \cap \tilde{\widetilde{B}}=\left\{\int_{x \in X} \mu_{\tilde{\tilde{A}}}(x) \cap \mu_{\tilde{\tilde{B}}}(x) / x\right\}= \\
& \left\{\int_{x \in X}\left[\begin{array}{c}
\int_{\alpha \in\left[\underline{\mu}_{\tilde{A}(x)} \wedge \underline{\mu}_{\tilde{B}(x)}, \bar{\mu}_{\tilde{A}(x)} \wedge \bar{\mu}_{\tilde{B}(x)}\right.} 1 / \alpha \\
\end{array}\right] / x\right\}
\end{aligned}
$$

The complement for interval type-2 fuzzy sets $\tilde{A}$ and $\tilde{B}$ is:

$$
-\tilde{\tilde{A}}=\left\{\int_{x \in X} \mu_{-\tilde{\tilde{A}}}(x) x\right\}=\int_{x \in X}\left[\underset{\alpha \in\left[1-\bar{\mu}_{\tilde{A}}(x), 1-\underline{\mu}_{\tilde{A}}(x)\right.}{\int_{1 / 2} 1 / \alpha}\right] / x
$$

In practice the computations in an IT2 FLS can be significantly simplified. Consider the rule base of an IT2 FLS consisting of $\mathrm{N}$ rules assuming the following form [22]:

$\mathrm{R}^{\mathrm{n}}$ : if $x_{1}$ is $X_{1}^{n}$ and $\ldots$ and $x_{i}$ is $X_{i}^{n}$, then $y$ is $Y^{n} ; n=1,2 \ldots N$.

where $X_{i}^{n} \quad(\mathrm{i}=1 \sim \mathrm{I})$ are IT2 fuzzy sets, and $Y^{n}=$ $\left[y_{1}^{n}, y_{2}^{n}\right]$ is an interval, which can be understood as the centroid $[9,24]$ of a consequent interval type-2 fuzzy set, or the simplest TSK model, for its simplicity. In many applications we use $y_{1}^{n}=y_{2}^{n}$, i.e., each rule consequent is a crisp number.

Assume the input vector is $x^{\prime}=\left(x_{1}^{\prime}, x_{2}^{\prime}, \ldots, x_{I}^{\prime}\right)$. Typical computations in an IT2FLS involve the 
following steps:

(1) Compute the membership of $\mathrm{x}_{i}^{\prime}$ on each $\mathrm{X}_{i}^{n}$

(2) Compute the firing interval of the $\mathrm{n}^{\text {th }}$ rule, $\mathrm{F}^{\mathrm{n}}\left(\mathbf{x}^{\prime}\right)$

(3) Perform type-reduction to combine $\mathrm{F}^{\mathrm{n}}\left(\mathbf{x}^{\prime}\right)$ and the corresponding rule consequents with the center-of-sets type-reducer [9]:

$$
Y_{\cos }\left(x^{\prime}\right)=\bigcup_{\substack{f^{n} \in F^{n}\left(x^{\prime}\right) \\ y^{n} \in Y^{n}}} \frac{\sum_{n=1}^{N} f^{n} y^{n}}{\sum_{n=1}^{N} f^{n}}=\left[y_{l}, \quad y_{r}\right]
$$

(4) Compute the defuzzified output as:

$$
y=\frac{y_{l}+y_{r}}{2}
$$

\subsection{Membership Functions}

Similar to type-1 fuzzy interpolation system, the input membership functions for both $x$ and $y$ directions and the predefined output membership functions for IT2FLS are shown in Fig. 5. The predefined output membership functions are used as default functions, and the final output membership function will be obtained by shifting the default those by the actual error values on the grid points.

We use $\mathrm{W}$ and $\mathrm{E}$ to represent the location of inputs in $x$ direction, $\mathrm{N}$ and $\mathrm{S}$ to the location of inputs in $y$ direction. For real outputs, four membership functions, $\mathrm{p}_{\mathrm{x} 1} \sim \mathrm{p}_{\mathrm{x} 4}$, should be designed for the $x$ direction, and another four membership functions, $p_{\mathrm{y} 1} \sim \mathrm{p}_{\mathrm{y} 4}$, are to be
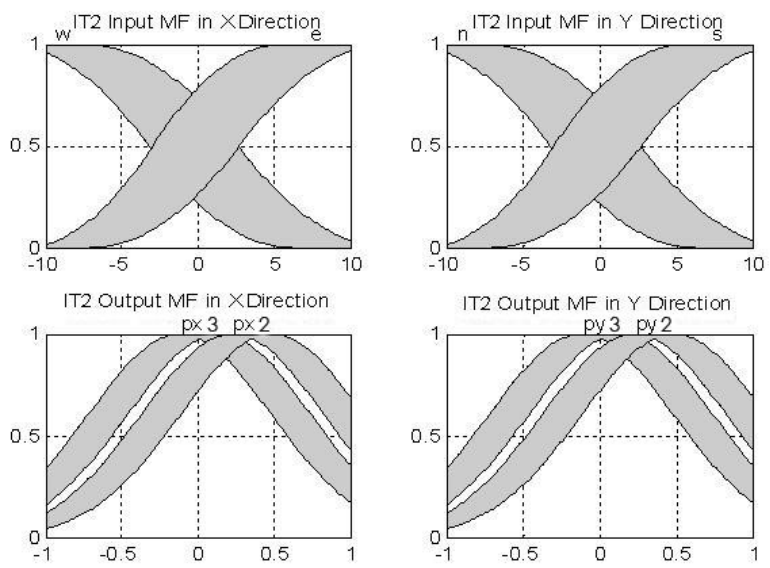

Fig. 5 The input and output membership functions for IT2 FLS. built for the $y$ direction. These output functions should be located at the center position, which are defined as the default location, as the beginning and changed to the real location based on the actual position errors on each grid point. In Fig. 5, these functions are all displayed but not in the default locations.

As for the control rules, the identical control rules are used for this IT2FLS, but the fuzzy sets are used as the degrees to replace those crisp values used in the type-1 FLS.

\section{Simulation Result}

Extensive simulation has been performed in order to illustrate the effectiveness of the proposed dynamic online IT2 fuzzy error interpolation technique in comparison to the type-1 FLS. Due to the random nature of the position errors, three different types of error are simulated in this study. These are:

- Normal distributed random error;

- Uniform distributed random error;

- Sinusoidal waveform error.

Figs. 6-8 show the simulation results of the type-1 and the IT2 fuzzy error interpolation techniques for these three types of error [24].

In these figures, the simulated target (testing) positions on the standard calibration board are spaced from $1 \mathrm{~mm}$ to $20 \mathrm{~mm}$ within each cell being with a size of $1 \mathrm{~mm}$.

Figs. 9-11 show comparisons in mean error, maximum error and STD values between type-1 and IT2 fuzzy error interpolation techniques in the histograms.

It can be seen that both mean errors and maximum errors of the IT2 fuzzy error interpolation technique are smaller than those of type-1 FLS methods. For all three error distribution, the mean errors of the IT2 fuzzy error interpolation method are approximately $10 \%$ to $20 \%$ smaller compared with those of type- 1 FLS method.

The maximum errors of the IT2 fuzzy error interpolation technique are about $10 \%$ to $30 \%$ smaller 

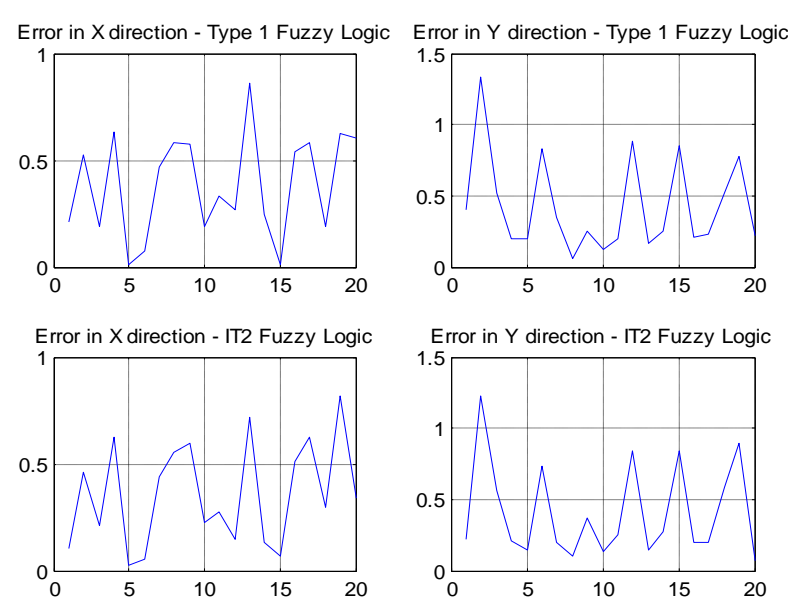

Fig. 6 Normal distributed random noise.
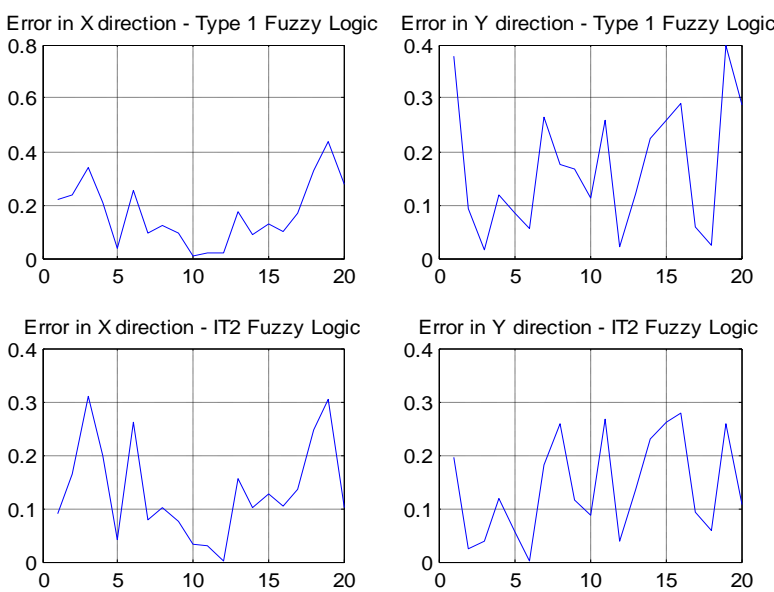

Fig. 7 Uniform distributed noise.
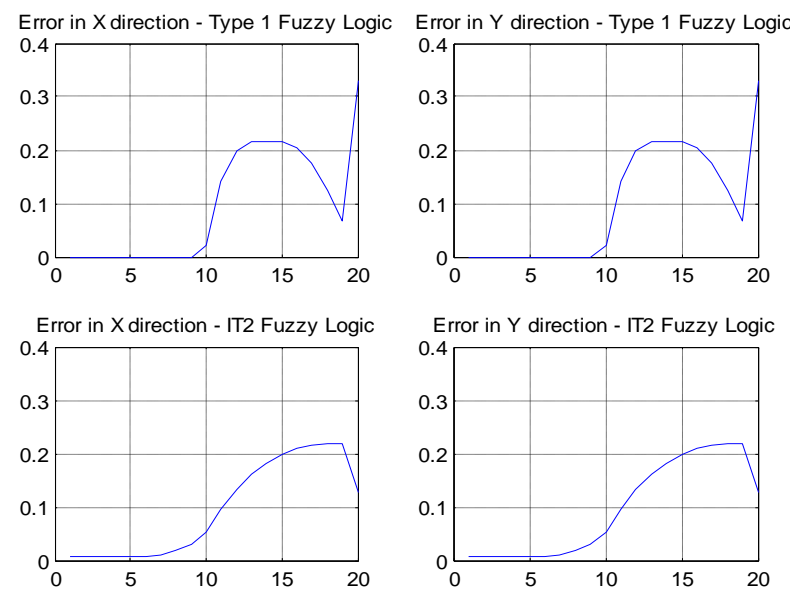

Fig. 8 Sinusoidal waveform noise.

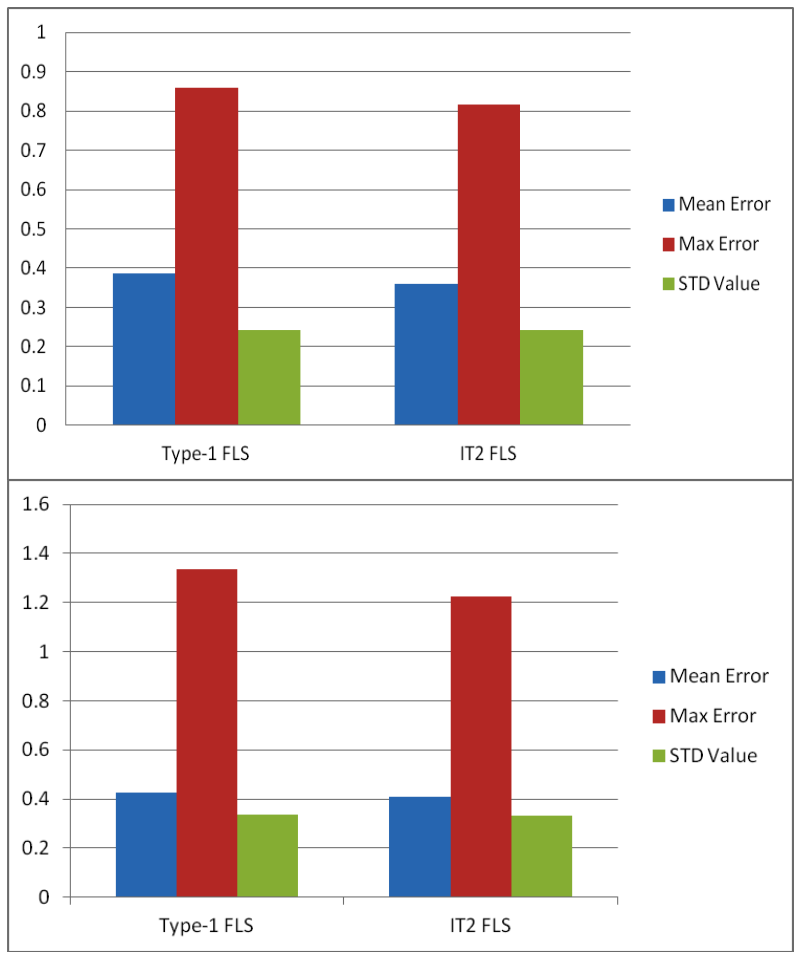

Fig. 9 Normal distributed random errors in $x$ and $y$ directions.

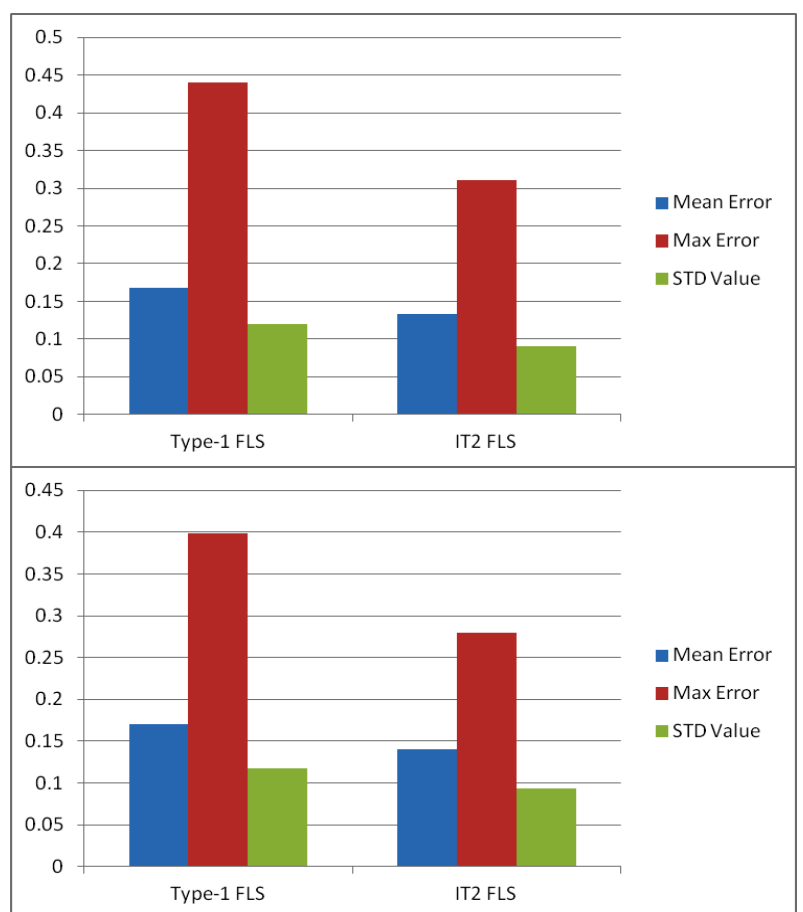

Fig. 10 Uniform distributed random errors in $x$ and $y$ directions. 


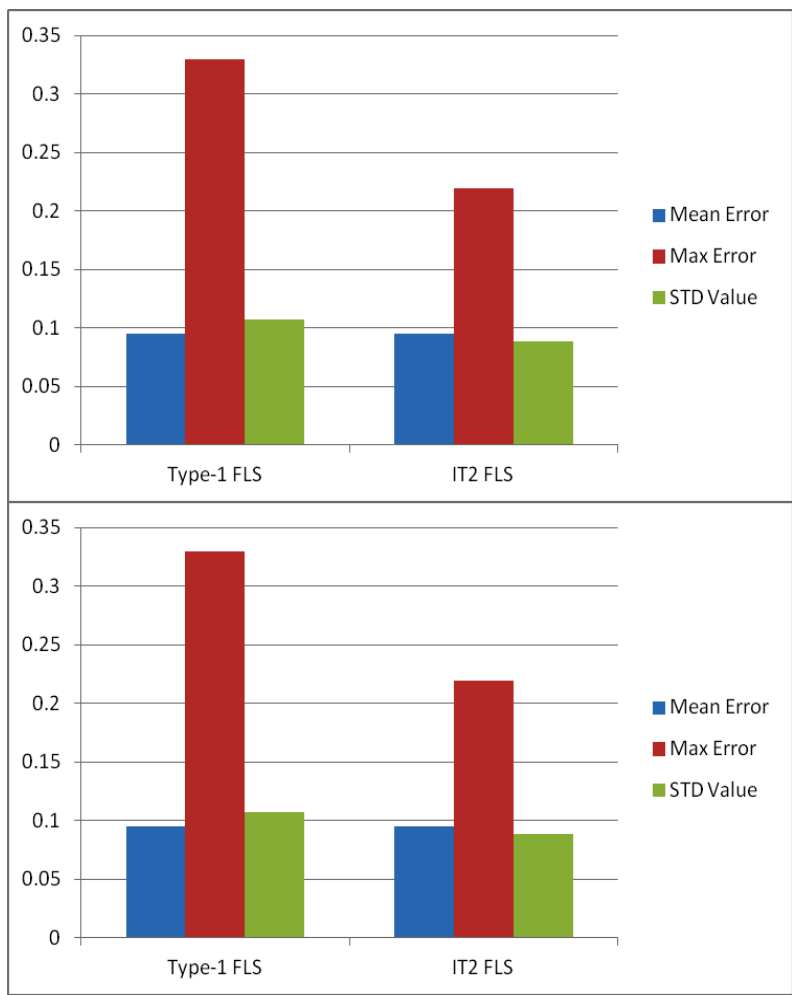

Fig. 11 Sinusoid distributed random errors in $x$ and $y$ directions.

than those of the type-1 FLS method. In one case (normal distribution error in $x$ and $y$ direction), the maximum errors of the IT2 fuzzy interpolation method are about $4 \%$ smaller than those of the type-1 FLS method.

Fig. 12 shows the IT2 fuzzy interpolation error surface.

The simulated results show the effectiveness of the dynamic on-line interval type-2 fuzzy error interpolation technique in reducing the position errors in the modeless robot compensation process.

To implement this interval type-2 fuzzy error interpolation technique as a real time application, an interface between the MATLAB ${ }^{\circledR}$ and high level programming languages $\mathrm{C} / \mathrm{C}++$ has been developed [25]. Although the most popular real time control programming language is $\mathrm{C} / \mathrm{C}++$, the fuzzy error interpolation method is developed and implemented in MATLAB [23]. Using this interface, the measured position errors on the grid points can be passed from $\mathrm{C} / \mathrm{C}++$ to MATLAB functions that implement the

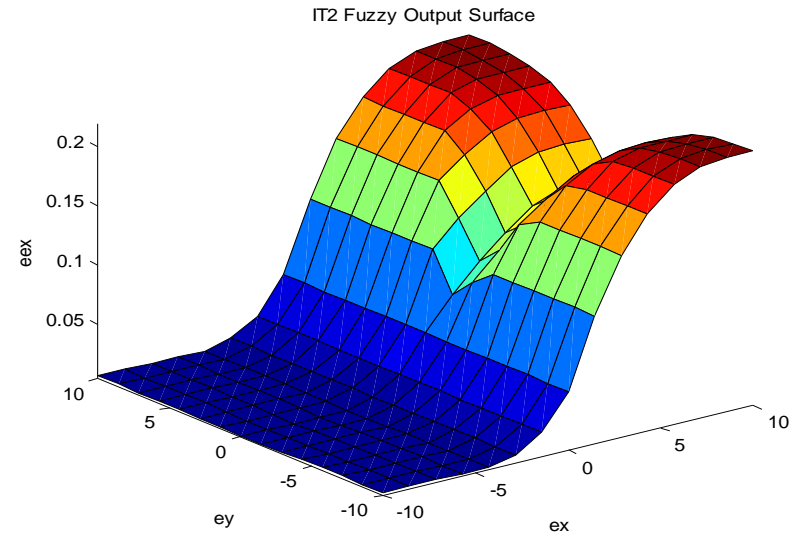

Fig. 12 The IT2 fuzzy interpolation error surface.

interval type-2 fuzzy error interpolation functions; and the fuzzy error interpolation results can be sent back to $\mathrm{C} / \mathrm{C}++$ for the real time controller to operate on the next target position.

\section{Conclusion and Summary}

A dynamic on-line interval type-2 fuzzy error interpolation technique is presented in this paper. The compensated position errors in a modeless robot calibration can be greatly reduced by the proposed technique. Simulation results demonstrate the effectiveness of the proposed fuzzy error interpolation technique. Three typical error models are utilized for comparison and simulation; these include sinusoidal waveform, normally distributed and uniformly distributed errors. This fuzzy error interpolation technique is ideal for the modeless robot position compensation, especially the high accuracy robot calibration process.

\section{Acknowledgment}

Special thanks to Dr. Oscar Castillo and his group to provide me the Interval Type-2 Fuzzy Logic Toolbox to enable us to perform our simulations for this paper $[26,27]$. We could not develop and share this paper with all of you without his sincere help and support.

\section{References}

[1] Mooring, B. W., Roth, Z. S., and Driels, M. R. 1991. 
Fundamentals of Manipulator Calibration. New York: John Wiley \& Sons.

[2] Penna, M. A. 1991. "Camera Calibration: A Quick and Easy Way to Determine the Scale Factor." IEEE Transactions on Pattern Analysis and Machine Intelligence 13: 1240-5.

[3] Weng, J., Cohen, P., and Herniou, M. 1992. "Camera Calibration with Distortion Models and Accuracy Evaluation." IEEE Transactions on Pattern Analysis and Machine Intelligence 14: 965-80.

[4] Du, F., and Brady, M. 1993. "Self Calibration of the Intrinsic Parameters of Cameras for Active Vision Systems." In Proceedings of the IEEE Conference on Computer Vision and Pattern Recognition, 477-82.

[5] Zhuang, H., and Roth, Z. S. 1996. Camera-Aided Robot Calibration. Florida: CRC Press, Inc.

[6] Triantafilis, J., Ward, W. T., Odeh, I. O. A, and McBratney, A. B. 2001. "Creation and Interpolation of Continuous Soil Layer Classes in the Lower Namoi Valley." Soil Science Society of America Journal 65 (2): 403-14.

[7] Suzana, D., Marceau, J. D., and Claude, M. 2001. "Space, Time, and Dynamics Modeling in Historical GIS Databases: A Fuzzy Logic Approach." Environment \& Planning B: Planning \& Design 28 (4): 545-63.

[8] Song, F., Smith, S. M., and Rizk, C. G. 1999. "A Fuzzy Logic Controller Design Methodology for 4D System with Optimal Global Performance Using Enhanced Cell State Space Based Best Estimate Directed Search Method." In Proceedings of the IEEE International Conference On Systems, Man and Cybernetics.

[9] Mendel, J. M. 2001. Uncertain Rule-Based Fuzzy Logic Systems: Introduction and New Directions. New Jersey: Prentice Hall PTR.

[10] Castillo, O., and Melin, P. 2008. Type-2 Fuzzy Logic: Theory and Applications (Studies in Fuzziness and Software Computing). Berlin:Springer.

[11] Aminifar, S., and Marzuki, A. 2013. "Uncertainty in Interval Type-2 Fuzzy Systems.” Mathematical Problems in Engineering, 16.

[12] Chang, Y. C. 2008. "A New Fuzzy Interpolative Reasoning Method Based on Interval Type-2 Fuzzy Sets." In Proceedings of the IEEE International Conference on Systems, Man and Cybernetics, 82-7.

[13] Kashyap, S. K. 2015. "IR and Color Image Fusion Using Interval Type 2 Fuzzy Logic System." In Proceedings of the International Conference on Cognitive Computing and Information Processing, 1-4.

[14] Lu, Q., Shi, P., Lam, H. K., and Zhao, Y. 2015. "Interval Type-2 Fuzzy Model Predictive Control of Nonlinear
Networked Control Systems." IEEE Transactions on Fuzzy Systems 99: 1-8.

[15] Wu, D., and Mendel, J. M. 2014. "Designing Practical Interval Type-2 Fuzzy Logic Systems Made Simple.” In Proceedings of the IEEE International Conference on Fuzzy Systems, 800-7.

[16] Schrieber, M., and Biglarbegian, M. 2014. "Hardware Implementation of a Novel Inference Engine for Interval Type-2 Fuzzy Control on FPGA." In Proceedings of the IEEE International Conference on Fuzzy Systems, 640-6.

[17] Tsai, C. C., Kao, J. C., and Chang, C. F. 2014. "Interval Type-2 Fuzzy Gear-Changing Control for Intelligent Bikes." In Proceedings of the International Conference on Machine Learning and Cybernetics, 741-7.

[18] Nurmaini, S., and Tutuko, B. 2014. "Motion Coordination for Swarm Robots." In Proceedings of the International Conference on ICT for Smart Society, 312-5.

[19] Mendel, J. M., Hagras, H., Tan, W. W., Melek, W. W., and Ying, H. 2014. Analytical Structure of Various Interval Type-2 Fuzzy PI and PD Controllers. Wiley-IEEE Press.

[20] Kumbasar, T. 2014. "Robust Stability Analysis of PD Type Single Input Interval Type-2 Fuzzy Control Systems." In Proceedings of the IEEE International Conference on Fuzzy System, 634-9.

[21] Zhuang, H., and Wu, X. M. 2001. "Membership Function Modification of Fuzzy Logic Controllers with Histogram Equalization." IEEE Transactions on Systems, Man, and Cybernetics, Part B: Cybernetics 31 (1).

[22] Wu, D. 2012. "A Brief Tutorial on Interval Type-2 Fuzzy Sets and Systems." Ph.D. thesis, University of Southern California.

[23] ITT/UABC 2008. Interval Type-2 Fuzzy Logic Toolbox For Use with MATLAB ${ }^{\circledR}$. Tijuana Institute of Technology and Baja California Autonomous University, Tijuana Campus, Mexico.

[24] Karnik, N. N., and Mendel, J. M. 2001. "Centroid of a Type-2 Fuzzy Set.” Information Sciences 132: 195-220.

[25] Bai, Y. 2003. Applications Interface Programming Using Multiple Languages. New Jersey: Prentice Hall PTR.

[26] Castro, J. R., Castillo, O., Melin, P., Martínez, L. G., Escobar, S., and Camacho, I. 2007. "Building Fuzzy Inference Systems with the Interval Type-2 Fuzzy Logic Toolbox." Analysis and Design of Intelligent Systems using Soft Computing Techniques 41: 53-62.

[27] Castro, J. R., Castillo, O., and Melin, P. 2007. “An Interval Type-2 Fuzzy Logic Toolbox for Control Applications." In Proceedings of the IEEE International Conference on Fuzzy System. 